\title{
Orexin/hypocretin receptor gene (HCRTR1) variation is associated with aggressive behaviour
}

Jaanus Harro ${ }^{1}$, Kariina Laas ${ }^{1}$, Diva Eensoo², Triin Kurrikoff ${ }^{3}$, Katre Sakala ${ }^{2,4}$, Mariliis Vaht $^{1}$, Jüri Parik ${ }^{5}$, Jarek Mäestu' ${ }^{6}$ Toomas Veidebaum ${ }^{4}$

${ }^{1}$ Division of Neuropsychopharmacology, Department of Psychology, University of Tartu, Tartu, Estonia

${ }^{2}$ Department of Family Medicine and Public Health, University of Tartu, Tartu, Estonia

${ }^{3}$ Division of Sociology, Department of Social Studies, University of Tartu, Tartu, Estonia

${ }^{4}$ National Institute for Health Development, Tallinn, Estonia

${ }^{5}$ Institute of Molecular and Cell Biology, University of Tartu, Tartu, Estonia

${ }^{6}$ Institute of Exercise and Sports Sciences, University of Tartu, Tartu, Estonia

Correspondending author. Division of Neuropsychopharmacology, Department of Psychology, University of Tartu, Estonian Centre of Behavioural and Health Sciences, Ravila 14A Chemicum, 50411 Tartu, Estonia. Email: Jaanus.Harro@ut.ee (J. Harro).

https://doi.org/10.1016/j.neuropharm.2019.02.009

Received 17 October 2018; Received in revised form 11 January 2019; Accepted 6 February 2019 Available online 10 February 2019 


\section{Highlights}

- Orexins are involved in the regulation of stress response

- The HCRTR1 rs2271933 G>A genotype was associated with aggressiveness

- A/A homozygotes were more aggressive and relapsed to drunk driving

- A-allele carriers were more frequently involved in traffic accidents

- The genotype effect is sensitive to stressful life events

Keywords: orexins; HCRTR1; genotype; aggressiveness; antisocial behaviour

\section{Abstract}

Orexins, alternatively called hypocretins, are neuropeptides with crucial role in maintaining wakefulness. The orexin system is thought to mediate a coordinated defense response but thus far investigated from the flight, but never fight, response perspective. An HCRTR1 gene variant (rs2271933 G > A) leading to amino acid substitution (Ile408Val) has been associated with migraine and mood disorders. We genotyped, and assessed aggressive behaviour in both birth cohorts ( $\mathrm{n}=655$ and 583) of the Estonian Children Personality Behaviour and Health Study (ECPBHS). Measures of aggressiveness were collected at age 25 or 33 and data on stressful life events (SLE-s) at age 15. Violations of traffic law were monitored in the samples of the Estonian Psychobiological Study of Traffic Behaviour. In both birth cohorts of the ECPBHS, the HCRTR1 the A/A homozygotes reported higher aggression in both BussPerry Aggression Questionnaire and the Life History of Aggression Interview. With either measure of aggressiveness, the HCRTR1 genotype effect was dependent on experience of SLE, the highest level of aggressiveness increase by environment being found in female $\mathrm{A} / \mathrm{A}$ homozygotes. The HCRTR1 A/A homozygotes scored higher in the ANGER facet of the Affective Neuroscience Personality Scale, while such an effect on FEAR was found only in females. Male HCRTR1 A/A homozygotes were more likely to relapse into drunk driving of a 
passenger car, and in two independent samples the A-allele carriers were causing traffic accidents more often. Conclusively, self-report, interview, and traffic record data converge indicating that the HCRTR1 Ile408Val genotype is associated with aggressiveness and breach of law.

This article is part of the Special Issue entitled "Current status of the neurobiology of aggression and impulsivity”.

\section{Introduction}

Orexins or hypocretins are hypothalamic neuropeptides derived from prepro-orexin (Sakurai et al., 1998; de Lecea et al., 1998) that have been implicated in a large variety of physiological functions, most clearly in sleep/wake regulation (Sutcliffe and de Lecea, 2000; Schwartz and Kilduff, 2015) and food intake (Sutcliffe and de Lecea, 2000; Broberger and Hökfelt, 2001; Ferrario et al., 2016). Orexin neurons are located in the dorsomedial and lateral hypothalamus, in particular in perifornical and magnocellular lateral hypothalamic nucleus (Broberger et al., 1998). More recently, orexins have been proposed to be involved in a coordinated adaptive defence response, in particular its anxiety, cardiorespiratory and endocrine aspects (Johnson et al., 2012). Orexins modulate neural circuits implicated in the expression and extinction of fear memories (Flores et al., 2015). The orexin system is activated during the acute stress response, but the effects of chronic stress on the orexin system are thought to be highly variable (Sargin, 2018). As orexin neurons are activated by a large variety of stressors, a hypothesis has been put forward that orexin neurotransmission is particularly important in behavioural regulation during aversive conditions involving high arousal (Berridge et al., 2010). Suicide attempters with major depression were found to have lower CSF levels of orexin as compared to other patient groups (Brundin et al., 2007), indirectly suggesting that orexin is important in maintaining adaptive strategies that are sensitive to mood regulation. 
If the orexin peptides are crucial mediators of coordinated stress response, then the other side of the fight-flight response, the possible choice of fight and preference of confrontational and aggressive response over escape should also be examined (Dhabhar, 2018). In a unique study on epileptic patients the release of orexin A was monitored in amygdala and found to be increased while subjects reported higher levels of anger (Blouin et al., 2013). Otherwise little evidence appears to exist on the role of orexins in any aspects of aggressive behaviour. Aggressiveness is however an important component of coordinated adaptive response to challenges presented by the environment (Van Kampen, 2015) and different nuclei of the hypothalamus play a critical role in this response (Kruk, 2014). Administration of orexin A has been reported to elicit dose-dependent complex changes in mouse maternal behaviour, including, at high doses, reduction of maternal behaviour and aggression (D'Anna and Gammie, 2006). Otherwise, no evidence for a role of orexins in aggressive behaviour has previously been made available. Nevertheless, orexin neurons project to such brain regions as septum and locus coeruleus that have a wellknown role in aggressiveness, and into emotion intergrative areas such as amygdala and the bed nucleus of stria terminalis. These brain regions also express type 1 orexin receptors (Flores et al., 2015).

Polymorphism of the orexin prepropeptide gene seems rather limited in humans, but several polymorphisms have been identified in the HCRTR1 and HCRTR2 genes encoding their receptors (Thompson et al., 2014). One HCRTR1 gene variant (rs2271933, G1222A) in exon 7 leads to amino acid substitution (Ile408Val) (Meerabux et al., 2005). This variant has been associated with migraine (Rainero et al., 2011b) and mood disorders (Rainero et al., 2011b). In migraine patients, aggressive behaviour is commonly observed, and their aggressiveness strongly correlates with symptoms of depression (Robbins, 2013; Park and Seo, 2018). Hence we sought to determine, by use of this marker, whether variation in the HCRTR1 gene can be associated with aggressiveness in two independent samples. Furthermore, taking advantage of 
a unique database of traffic behaviour, a common behaviour that may include breach of law that is objectively recorded, we studied the association of the HCRTR1 genotype with drunk driving and involvement in accidents in two samples.

\section{Material and methods}

\subsection{Subjects}

\subsubsection{The longitudinal Estonian Children Personality Behaviour and Health Study}

Assessment of aggressiveness in this investigation has been carried out on two independent samples included in the Estonian Children Personality Behaviour and Health Study (ECPBHS). We used its original EYHS subsample that comprises two birth cohorts. At recruitment the children were at either third (younger cohort, average age 9.6 years, 52.3\% female) or ninth (older cohort, average age 15.6 years, 56.2\% female) grade; the participation rate was $79.1 \%$. The rationale of sample selection and procedure has been described previously (Harro et al., 2001), and the recent data collection waves up to age 25 have been described in more detail elsewhere (e.g., Kiive et al., 2017; Laas et al., 2017). Data on aggressiveness and affective aspects of personality were collected in the most recent data collection waves, i.e., at age 25 for the younger cohort and age 33 for the older cohort. The number of participants was 441 and 504, respectively. All participants signed an informed consent form. The ECPBHS has been approved by the Tartu University Ethics Review Committee on Human Research.

\subsubsection{Samples of the traffic studies}

We also genotyped the subjects of two longitudinally observed samples from the Estonian Psychobiological Study on Traffic Behaviour (EPSTB) in order to examine the association of the HCRTR1 genotype with objectively recorded antisocial behaviour. The EPSTB Sample 1 comprised subjects recruited in studies of drunk drivers and speed limit exceeders (see 
Eensoo et al., 2005 and Paaver et al., 2006 for detailed description). This group of drivers included male subjects who were identified by the police driving drunk at least once during the previous year ( $n=203$; mean age \pm SD, $33 \pm 11$ years) or exceeding speed limits $(n=292$; mean age $36 \pm 11$ years). The control group was formed by computerised random choice of the male subjects in the driving licence database of the Estonian Motor Vehicle Registration Centre; it consisted of 509 individuals, with a mean age of $37 \pm 12$ years. Subjects were contacted by telephone, the description and aims of the study were provided, and they filled in self-reported questionnaires during a visit to laboratory where they donated venous blood samples. The EPSTB Sample 2 was formed during an impulsivity-focused intervention study at traffic schools (Paaver et al., 2013; Eensoo et al., 2018). Of the 1886 participants (mean age $23.0 \pm 7.2$ years) of the intervention study, altogether 767 subjects ( $41 \%$ male) had agreed to donate a blood sample. All participants signed an informed consent form. This study was approved by the Tartu University Ethics Review Committee on Human Research.

\subsection{Genotyping HCRTR1 rs2271933}

Genomic DNA was extracted from venous blood samples using Qiagen QIAamp ${ }^{\circledR}$ DNA Blood Midi Kit. The real-time polymerase chain reaction (RT-PCR) for genotyping the HCRTR1 rs2271933 polymorphism was performed using a TaqMan Pre-Designed SNP Genotyping Assay (Applied Biosystems; Foster City, CA, USA) C_115961465_10 containing primers and fluorescent probes. Genotyping reactions were performed in a total volume of $10 \mu \mathrm{l}$ with $\sim 25 \mathrm{ng}$ of template DNA. RT-PCR reaction components and final concentrations were as follows: 1:5 5 x HOT FIREPol ${ }^{\circledR}$ Probe qPCR Mix Plus (ROX) (Solis BioDyne) and 1:20 80 x TaqMan Primers Probe. Context sequence [VIC/FAM] was as follows:

CTTGTCCTTGCAGAGCCGATGCTCC[A/G]TCTCCAAAATCTCTGAGCATGTGGT. Reactions were performed on the Applied Biosystems ViiA TM 7 Real-Time PCR System. The 
amplification procedure consisted of an initial denaturation step at $95{ }^{\circ} \mathrm{C}$ for $12 \mathrm{~min}$ and 40 cycles of $95{ }^{\circ} \mathrm{C}$ for $15 \mathrm{~s}$ and $60{ }^{\circ} \mathrm{C}$ for $1 \mathrm{~min}$. Positive and negative controls were added to each reaction plate. No inconsistencies occurred. Genotyping was performed blind to all phenotypic data. Allele frequencies agreed with National Centre for Biotechnology Information database (minor allele frequency around 0.4; https://www.ncbi.nlm.nih.gov/snp/rs2271933\#frequency tab) and published reports. All the subjects were of European origin, and genotype frequencies of all samples were in HardyWeinberg equilibrium (Table 1).

Table 1. HCRTR1 rs2271933 genotype frequencies in samples included in this investigation.

\begin{tabular}{|l|c|c|c|c|}
\hline & $\mathrm{A} / \mathrm{A}$ & $\mathrm{A} / \mathrm{G}$ & $\mathrm{G} / \mathrm{G}$ & Total \\
\hline ECPBHS total & $185(15 \%)$ & $610(50 \%)$ & $439(35 \%)$ & $1234(100 \%)$ \\
\hline $\begin{array}{l}\text { ECPBHS younger } \\
\text { cohort }\end{array}$ & $75(13 \%)$ & $309(53 \%)$ & $196(34 \%)$ & $580(100 \%)$ \\
\hline $\begin{array}{l}\text { ECPBHS older } \\
\text { cohort }\end{array}$ & $110(17 \%)$ & $301(46 \%)$ & $243(37 \%)$ & $654(100 \%)$ \\
\hline EPSTB Sample 1 & $160(16 \%)$ & $467(48 \%)$ & $357(36 \%)$ & $984(100 \%)$ \\
\hline EPSTB Sample 2 & $132(17 \%)$ & $371(48 \%)$ & $273(35 \%)$ & $776(100 \%)$ \\
\hline
\end{tabular}

Minor allele frequency 0.40. For comparison, around 0.40 in European samples in the NCBI database; 0.367 in the 1000G CEU population (https://www.ncbi.nlm.nih.gov/snp/rs2271933\#frequency tab).

\subsection{Measures of aggressiveness}

\subsubsection{Self-report questionnaire}

The 29-item Buss-Perry Aggression Questionnaire (BPAQ; Buss and Perry, 1992) assesses four aspects of aggressive behaviour: Physical aggression, Verbal aggression, Anger, and Hostility. Participants rated each statement on a 5-point Likert Scale (uncharacteristic = 1, characteristic $=5$ ). Data of Buss-Perry Aggression Questionnaire were available for 427 subjects in the younger ECPBHS cohort and for 499 subjects in the older cohort.

\subsubsection{Aggressiveness interview}

The Life History of Aggression interview (LHA; Coccaro et al., 1997) was carried out by an experienced clinical psychologist. Items were scored only for the history of actual behaviour. 
LHA has three subscales: Aggression (temper tantrums, verbal aggression, indirect aggression, non-specific fighting, and physical assault against people);

Consequences/Antisocial Behaviour (school disciplinary problems, problems with supervisors, antisocial behaviour not resulting in police involvement, and antisocial behaviour involving the police); and Self-Directed Aggression (self-injurious behaviour, and suicide attempts). Each item was rated on a 5 -point scale, ranging from $0=$ No events to $5=$ More events than can be counted. Data of LHA were available for 427 subjects in the ECPBHS younger cohort and 495 subjects in the older cohort. Overall, Aggression assessed by interview was moderately correlated to measures of BPAQ self-reported aggressiveness in both sexes while Antisocial Behaviour was associated to self-reports moderately in males and weakly in females (Supplementary Table 1). Correlation of Self-directed Aggression with other measures was weak.

\subsection{Affective Neuroscience Personality Scale}

We used the adaptation of the short version of the ANPS (Davis et al., 2003) that is a selfreport instrument constructed bottom-up to correspond to the activity in neural circuits underlying basic emotive systems as defined in animal research (Panksepp, 1998; Davis and Panksepp, 2011). It comprises scales termed ANGER, FEAR, SADNESS, SEEKING, CARE and PLAY, each measured with six items, each on a 5-point scale. Data on ANPS were available for 423 subjects in the ECPBHS younger cohort and 502 subjects in the older cohort.

\subsection{Stressful life events}

Stress experienced in childhood was measured at age 15 with a list of 21 adverse life experiences reported by participants or their parents, which included questions about parental death and divorce/separation, absence of both parents, unemployment in the family, financial 
problems and poverty in the household, poor living conditions, poor health and chronic diseases, serious illness of a family member, death of a close relative, trauma, fear of school, bullying at school and humiliation at home (Laas et al., 2014). The events were recorded as dichotomous variables (present or not present) and were then counted to form the number of experienced stressful life events $\left(\mathrm{SLE}_{15}\right)$. The SLE distribution did not differ between males and females. Data were available for 478 subjects in the ECPBHS younger cohort and for 591 subjects in the older cohort.

\subsection{Database search}

Data on violations of traffic law and traffic accidents were obtained from databases maintained by the traffic police and the traffic insurance fund for the period of January 1 , 2002 to December 31, 2011 for the EPSTB Sample 1 and for the period of January 1, 2007 to December 31, 2011 for the EPSTB Sample 2 (Eensoo et al., 2018). We focused on driving while impaired by alcohol (DWI; penalties for drunk driving with an estimated blood alcohol level of $0.2 \%$ or more) and traffic accidents. The accidents where the subject was at fault were classified as active accidents, and other accidents as passive.

\subsection{Statistical analysis}

Data were analysed using IBM SPSS (version 22.0, Chicago, IL) and SAS (version 9.4 SAS Inc., Cary, NC) software. One- and two-way analyses of variance were performed to reveal differences between HCRTR1 genotypes with and without consideration of stressful life events (dichotomized by median split). When appropriate Tukey post-hoc tests were applied. We also used a series of multiple linear regressions for predicting the aggressiveness-related scores where we added all the simple terms and interaction terms to the models simultaneously. To increase interpretability and minimize collinearity in the models, predictors were centered according to Kraemer and Blasey (2004), and interaction terms were 
residuals-centered according to Little et al. (2006). Number of stressful life events were treated as quantitative variable for regression analysis. Models were evaluated by goodness of fit statistics and analyses of residuals. Chi-square tests were conducted to assess distribution of presence of traffic violations and accidents by genotype. The conventional 5\% level was applied, given that this is the first exploratory study on the association of the orexin receptor genotype and aggressiveness measures, and that the latter are significantly inter-correlated. Retrospective power calculations indicated that if the effects were small the power was low, too, starting from 0.584 in case of p-value 0.050 . However, in all analyses where the p-value was $\leq 0.008$, the power was adequate $(\geq 0.8)$.

\section{Results}

\subsection{HCRTR1 genotype and aggressiveness in the two population-representative birth}

\section{cohorts}

In the ECPBHS younger cohort aggressiveness was assessed at age 25. The HCRTR1 Ile408Val (G/A) genotype was significantly associated with total score of the Buss-Perry Aggression Questionnaire $\left[\mathrm{F}(2,424)=4.36 ; \mathrm{p}=0.013 ; \eta^{2}=0.020\right]$, the A/A homozygotes reporting higher hostility, anger and physical aggression (Fig. 1A and Supplementary Table 2). Similarly, the genotype was associated with the total score of the Lifetime History of Aggression interview $\left[\mathrm{F}(2,424)=6.98 ; \mathrm{p}=0.001 ; \eta^{2}=0.032\right]$ and its major subscale Aggression (Fig. 1B and Supplementary Table 2), the A/A homozygotes reporting higher level of aggressiveness.

Thereafter the ECPBHS older cohort was independently examined for aggressiveness at age 33. The findings were essentially similar: The total score of the BPAQ was significantly higher in the A/A homozygotes [F (2496] = 3.43; $\left.\mathrm{p}=0.033 ; \eta^{2}=0.014\right]$, with significant differences in anger and physical aggression (Fig. 2A and Supplementary Table 2). The 
HCRTR1 genotype was also significantly associated with the LHA Aggression subscale (Fig.

2B and Supplementary Table 2) as well as with self-directed aggression, while regarding total score the conventional level of statistical significance was missed $(p=0.06)$. Given the similarity of the genotype effect on these primary outcome measures in both cohorts, further stratified analyses used the data of the birth cohorts combined.
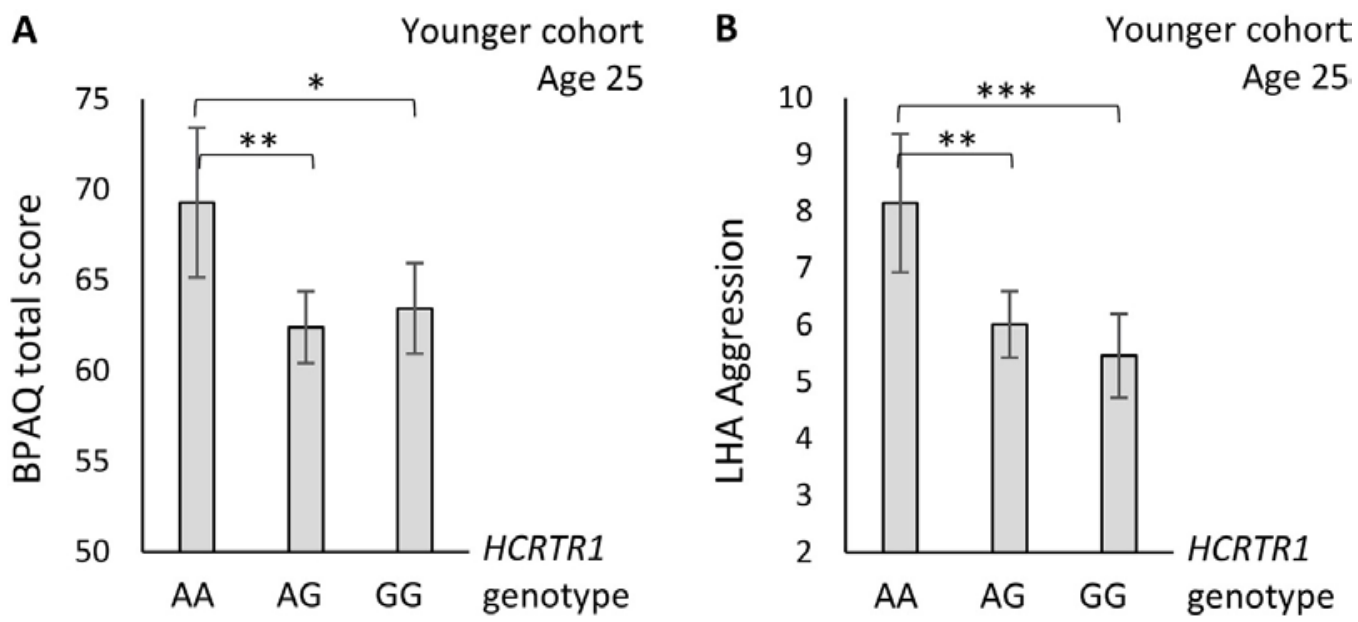

Fig. 1. HCRTR1 genotype and aggressiveness in the younger ECPBHS birth cohort at age 25. A Buss-Perry Aggression Questionnaire (BPAQ) total score; number of subjects $A / A=53, A / G=230, G / G=144$; $B$ - Life History of Aggression (LHA) interview Aggression subscale; number of subjects $A / A=53, A / G=230$, $\mathrm{G} / \mathrm{G}=144 .{ }^{*}-\mathrm{p}<0.05, * *-\mathrm{p}<0.01, * * *-\mathrm{p}<0.001$ difference between genotypes. Whiskers indicate $95 \%$ confidence intervals.

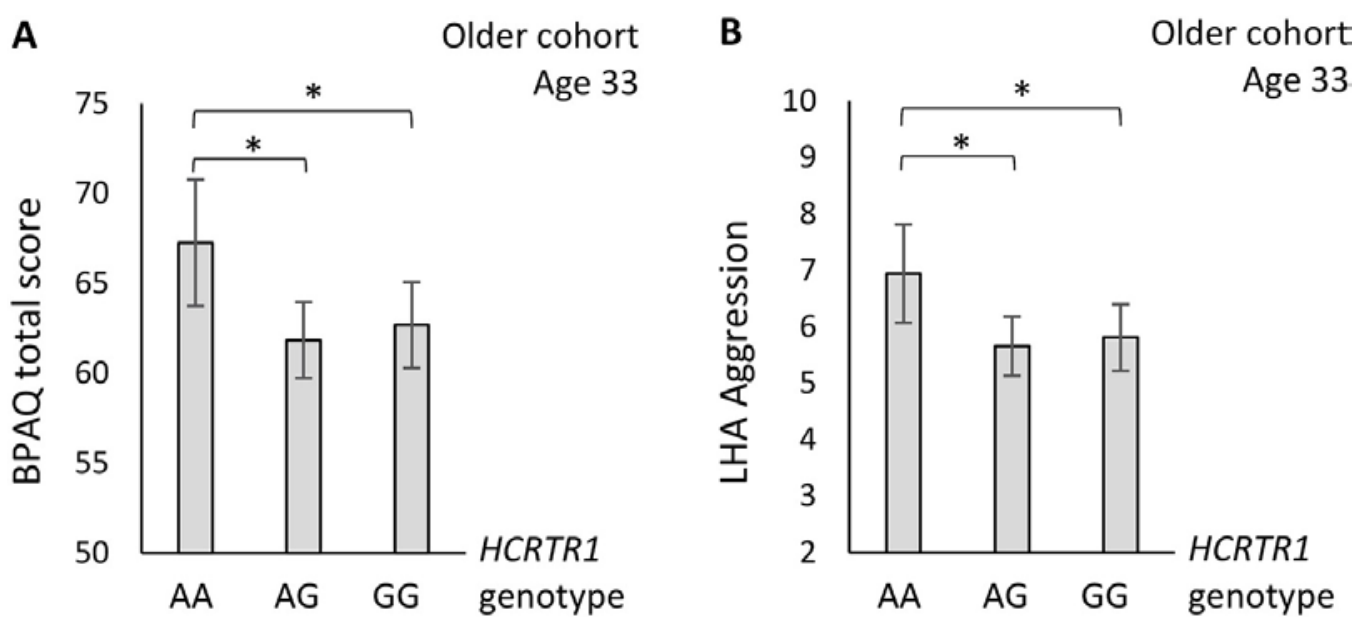

Fig. 2. HCRTR1 genotype and aggressiveness in the older ECPBHS birth cohort at age 33. A - BussPerry Aggression Questionnaire (BPAQ) total score; number of subjects A/A = 84, A/G = 232, G/G = 183; B Life History of Aggression (LHA) interview Aggression subscale; number of subjects $A / A=83, A / G=230$, $\mathrm{G} / \mathrm{G}=182 . *$ - $\mathrm{p}<0.05$ difference between genotypes. 
Analysis of both cohorts together revealed highly significant genotype effects on the BPAQ and LHA total scores and most aspects of aggressive behaviour (Supplementary Table 2). No genotype by sex interaction was statistically significant (data not shown).

\subsection{HCRTR1 genotype and the Affective Neuroscience Personality Scale}

Based on the findings above we developed an a priori hypothesis that the HCRTR1 genotype should be associated with scores of the ANGER scale, and given the literature on stress reactivity and orexin, possibly with the FEAR scale, as a large share of overall aggressiveness is of defensive type. Indeed we found that ANGER was significantly higher in the A/A homozygotes $\left[\mathrm{F}(2,927)=6.39 ; \mathrm{p}=0.002 ; \eta^{2}=0.014\right]$; this was the case in both males and females (Table 2). As to FEAR, the genotype main effect missed the conventional level of significance $\left[\mathrm{F}(2,927)=2.44 ; \mathrm{p}=0.088 ; \eta^{2}=0.005\right]$ and no statistically significant genotype $\times$ sex interaction emerged $[F(2,924)=2.36 ; p=0.095 ; \eta 2=0.005]$, but the scores were much higher in females, so we analysed the sexes separately. While in males the genotypes were identical $\left[\mathrm{F}(2,396)<0.01 ; \mathrm{p}=0.997 ; \eta^{2}<0.001\right]$, a variation appeared among females $\left[\mathrm{F}(2,396)=5.09 ; \mathrm{p}=0.006 ; \eta^{2}=0.019\right]$. The female A/A homozygotes had the highest levels of FEAR scores. Exploratory analysis of other affect domains (Table 2)

Table 2. HCRTR1 genotype and the Affective Neuroscience Personality Scale scores in both ECPBHS birth cohorts combined, by sex.

\begin{tabular}{lcccccc}
\hline & \multicolumn{3}{c}{ Males } & \multicolumn{3}{c}{ Females } \\
& AA & AG & GG & AA & AG & GG \\
\hline ANGER & $17.5 \pm 4.4$ & $15.9 \pm 4.4^{*}$ & $16.0 \pm 4.5^{*}$ & $18.2 \pm 4.5$ & $16.6 \pm 4.4^{*}$ & $17.1 \pm 5.0$ \\
FEAR & $15.7 \pm 3.3$ & $15.7 \pm 3.1$ & $15.8 \pm 3.0$ & $18.2 \pm 3.5$ & $16.8 \pm 3.3^{* *}$ & $17.4 \pm 3.3$ \\
SADNESS & $13.5 \pm 4.0$ & $13.1 \pm 4.1$ & $13.0 \pm 4.2$ & $16.2 \pm 4.8$ & $15.0 \pm 4.7$ & $15.6 \pm 5.0$ \\
& & & & & & $23.2 \pm 3.6$ \\
SEEKING & $23.5 \pm 2.9$ & $23.0 \pm 3.7$ & $22.7 \pm 3.6$ & $22.2 \pm 3.4^{\#}$ & $23.2 \pm 3.5$ & $23.2 \pm 3.5$ \\
CARE & $20.6 \pm 4.2$ & $20.0 \pm 3.9$ & $20.5 \pm 3.8$ & $23.1 \pm 3.0$ & $22.7 \pm 3.6$ & 22.6 \\
PLAY & $22.5 \pm 4.6$ & $22.1 \pm 3.9$ & $22.1 \pm 3.9$ & $21.9 \pm 4.0$ & $22.7 \pm 3.8$ & $22.6 \pm 4.5$ \\
\hline
\end{tabular}

Mean \pm S.D. ${ }^{*}-\mathrm{p}<0.05,{ }^{* *}-\mathrm{p}<0.01$, difference from A/A genotype in the same sex. ${ }^{*}-\mathrm{p}<0.05$, difference from the A/A male genotype. Genotype frequencies: Males, A/A = 61, A/G = 193, G/G = 145; Females, A/A = $74, \mathrm{~A} / \mathrm{G}=273, \mathrm{G} / \mathrm{G}=184$. 
revealed no main effect of genotype, but a genotype $\times$ sex interaction was revealed for

SEEKING $\left[\mathrm{F}(2,924)=3.25 ; \mathrm{p}=0.040 ; \eta^{2}=0.016\right]$. This was owing to the relatively higher SEEKING score of the A/A males contrasted to the relatively low SEEKING in A/A females (Table 2).

\subsection{HCRTR1 genotype and stressful life events (SLE): interaction effect on}

\section{aggressiveness}

Three-way ANOVA-s as well as multidimensional linear regression analysis suggested the presence of HCRTR1 genotype $\times$ SLE $\times$ sex interactions for the BPAQ and LHA measures (Supplementary Tables 3 and 4). Higher level of experience of stressful life events by age 15 had a general tendency, in some genotype-wise comparisons statistically significant, to increase aggressiveness, except in the group with highest aggressiveness, the male A/A homozygotes (Fig. 3). In females, higher exposure to SLE-s was in A/A homozygotes associated with a dramatic increase. This was the case for both questionnaire (BPAQ) and interview (LHA) measures.
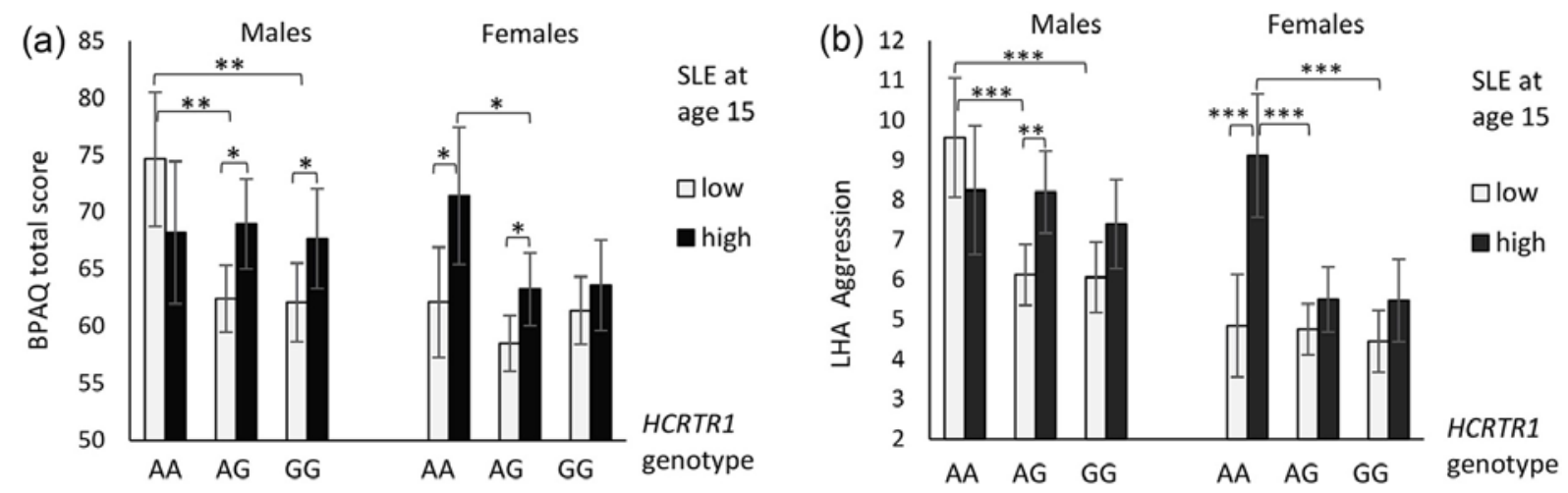

Fig. 3. Aggressiveness by HCRTR1 genotype, level of exposure to stressful life events and sex. Panel A: Buss-Perry Aggression Questionnaire. Number of males: Low SLE, A/A= 27, A/G = 107, G/G =79; High SLE, $A / A=24, A / G=60, G / G=49$. Number of females: Low SLE, $A / A=40, A / G=157, G / G=106$; High SLE, $A / A=26, A / G=92, G / G=59$. Panel B: Lifetime History of Aggression. Number of males: Low SLE, $A / A=28$, A/G=108, G/G=80; High SLE, AA=24, AG=59, GG =50. Number of females: Low SLE, $A / A=38, A / G=154$, G/G=103; High SLE, $A / A=26, A / G=93, G / G=59 . *-p<0.05, * *$ - $p<0.01$, *** $-p<0.001$ difference between genotypes. Whiskers indicate $95 \%$ confidence intervals. 


\subsection{HCRTR1 genotype and re-occurrence of driving while impaired}

Fig. 4 presents the occurrence of drunk driving during the observation period by year. While some cases of DWI appeared over the years in the random control group and among the speed limit exceeders, the subjects recruited owing to a recorded episode of drunk driving had the levels of relapse that far exceeded the prevalence of DWI in other groups. This reoccurrence of DWI was however related to the HCRTR1 genotype (Supplementary Table 5). Higher re-occurrence of DWI in the HCRTR1 A/A homozygotes appeared soon after initial recruitment, became statistically significant at the sixth year of follow-up ( $\mathrm{DF}=2, \chi^{2}=7.4$, $\mathrm{p}=0.025)$ and remained so until the last year of follow-up $\left(\mathrm{DF}=2, \chi^{2}=7.7, \mathrm{p}=0.022\right)$. At the recruitment, the drunk drivers, speed limit exceeders and controls were not different in terms of genotype distribution (data not shown).

\subsection{HCRTR1 genotype and involvement in traffic accidents}

We examined in this sample whether or not the HCRTR1 genotype could be related to involvement of the participants in traffic accidents. It was revealed that the accumulation of active traffic accidents in the DWI group was higher in the A-allele carriers (Fig. 5 and Supplementary Table 5). A tendency for this was present early in the observation period but as the cases accumulated this trend became statistically significant by the seventh year of observation $\left(\chi^{2}=4.2, p=0.041\right)$ and remained so until the last year examined $\left(\chi^{2}=5.0\right.$, $\mathrm{p}=0.025$ ). Similar findings were present in an independent sample of passenger car drivers observed since they entered the study (Fig. 6 and Supplementary Table 5). In this sample the number of the HCRTR1 A-alleles seemed associated with the involvement of traffic accidents, and in particular with active accidents, but statistical significance was found in comparison of either A/A homozygotes or A-allele carriers with G/G homozygotes. 


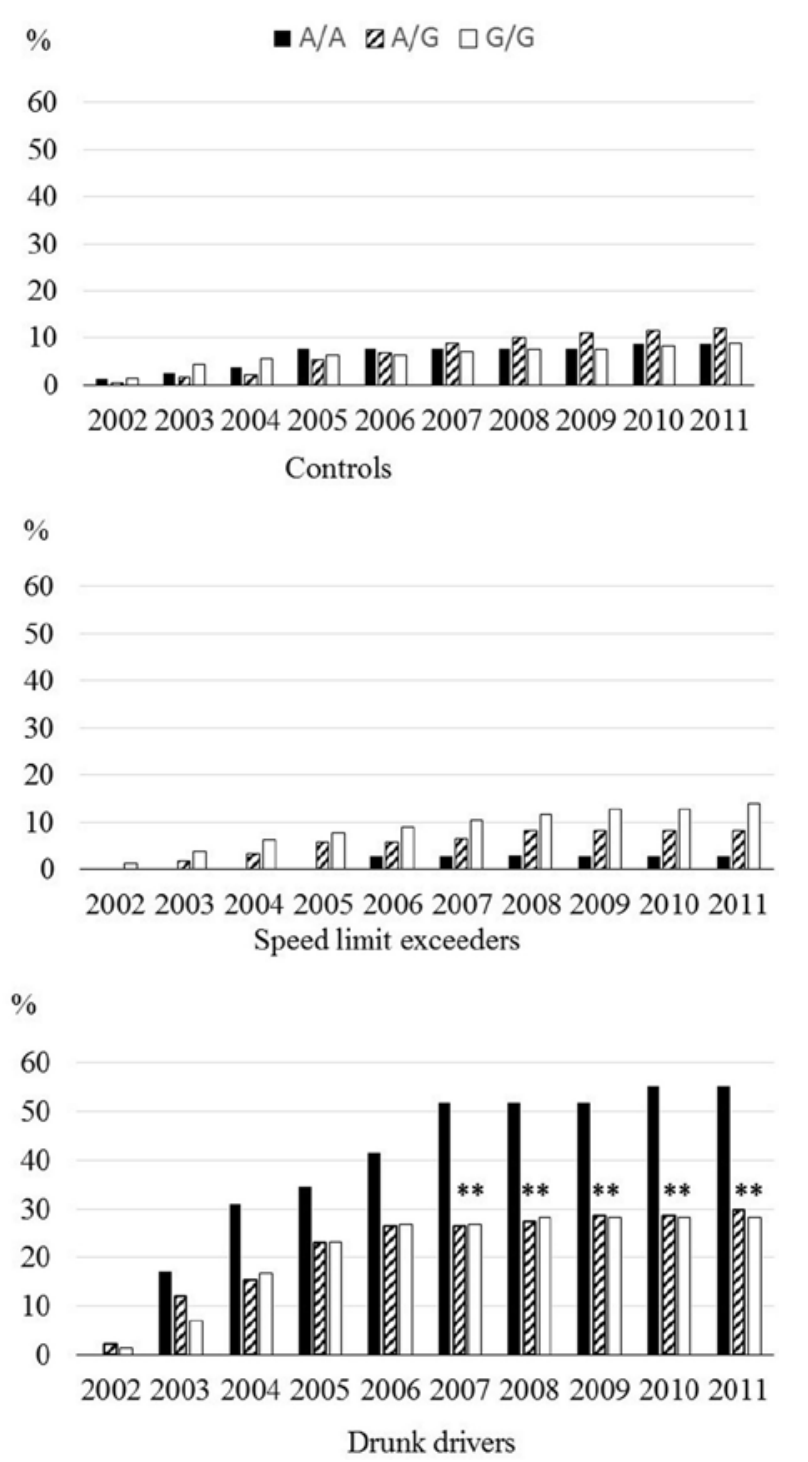

Fig. 4. Cumulative occurrence of driving while impaired in 2002-2011 by year in subjects recruited in 2001-2003, by the HCRTR1 genotype. The $\mathrm{Y}$-axis represents the proportion of subjects with the given genotype who had by that year driven vehicle while intoxicated. For the drunk drivers group this refers to re-occurrence. ${ }^{*}$ - $\mathrm{p}<$ 0.05 , difference from the A/A genotype. Genotype frequency: Controls, $A / A=80, A / G=190, G / G=$ 160; Speed limit exceeders, $A / A=34, A / G=121$, $\mathrm{G} / \mathrm{G}=78$; Drunk drivers $\mathrm{A} / \mathrm{A}=29, \mathrm{~A} / \mathrm{G}=91, \mathrm{G} / \mathrm{G}$ $=71$.
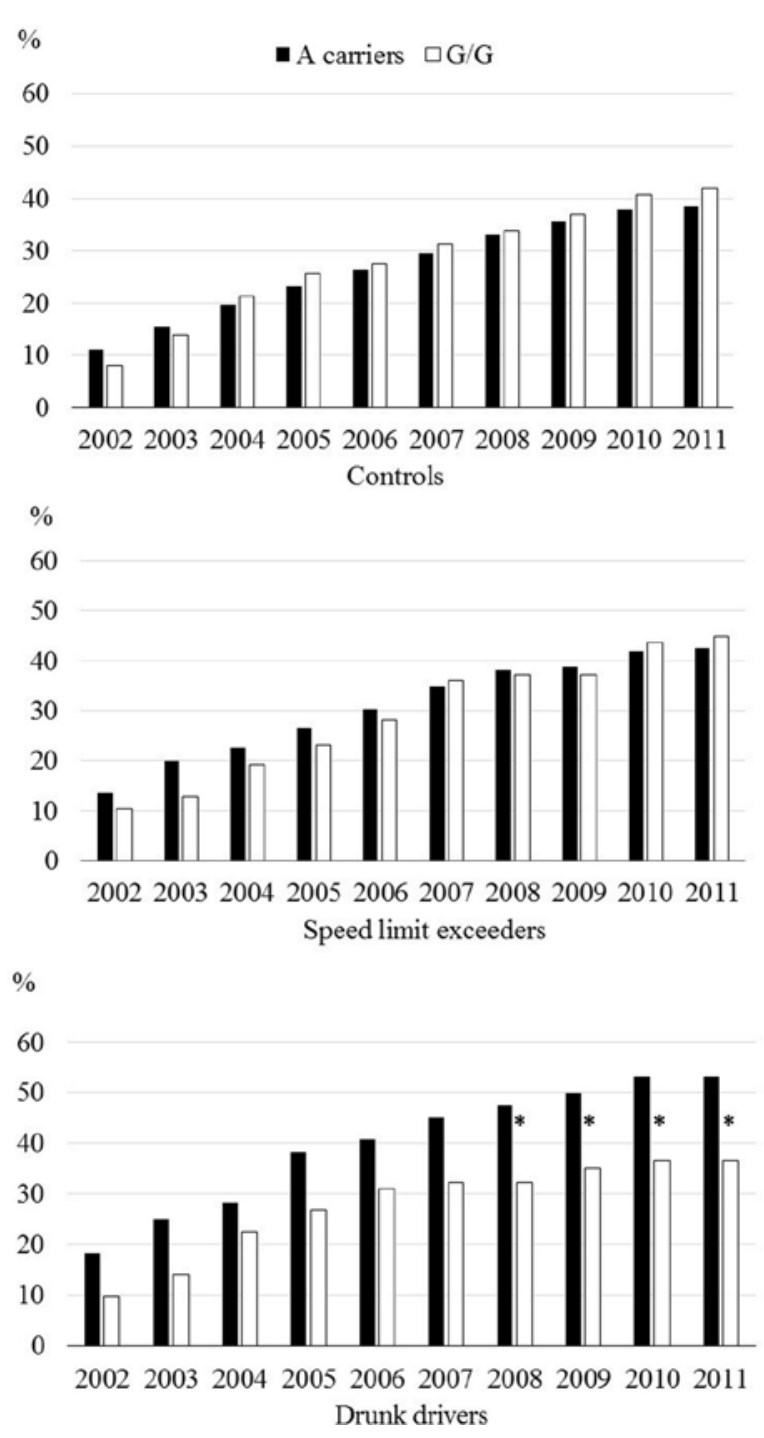

Fig. 5. Cumulative occurrence of active traffic accidents in 2002-2011 by year in subjects recruited in 2001-2003, by the HCRTR1 genotype. The Y-axis represents the proportion of subjects with the given genotype who had by that year caused a traffic accident. $*$ - $\mathrm{p}<0.05$, difference from the A-allele carriers. Genotype frequency: Controls, A-allele carriers $=270, \mathrm{G} / \mathrm{G}=$ 160; Speed limit exceeders, A-allele carriers $=151$, $\mathrm{G} / \mathrm{G}=78$; Drunk drivers, A-allele carriers $=120$ $\mathrm{G} / \mathrm{G}=71$. 

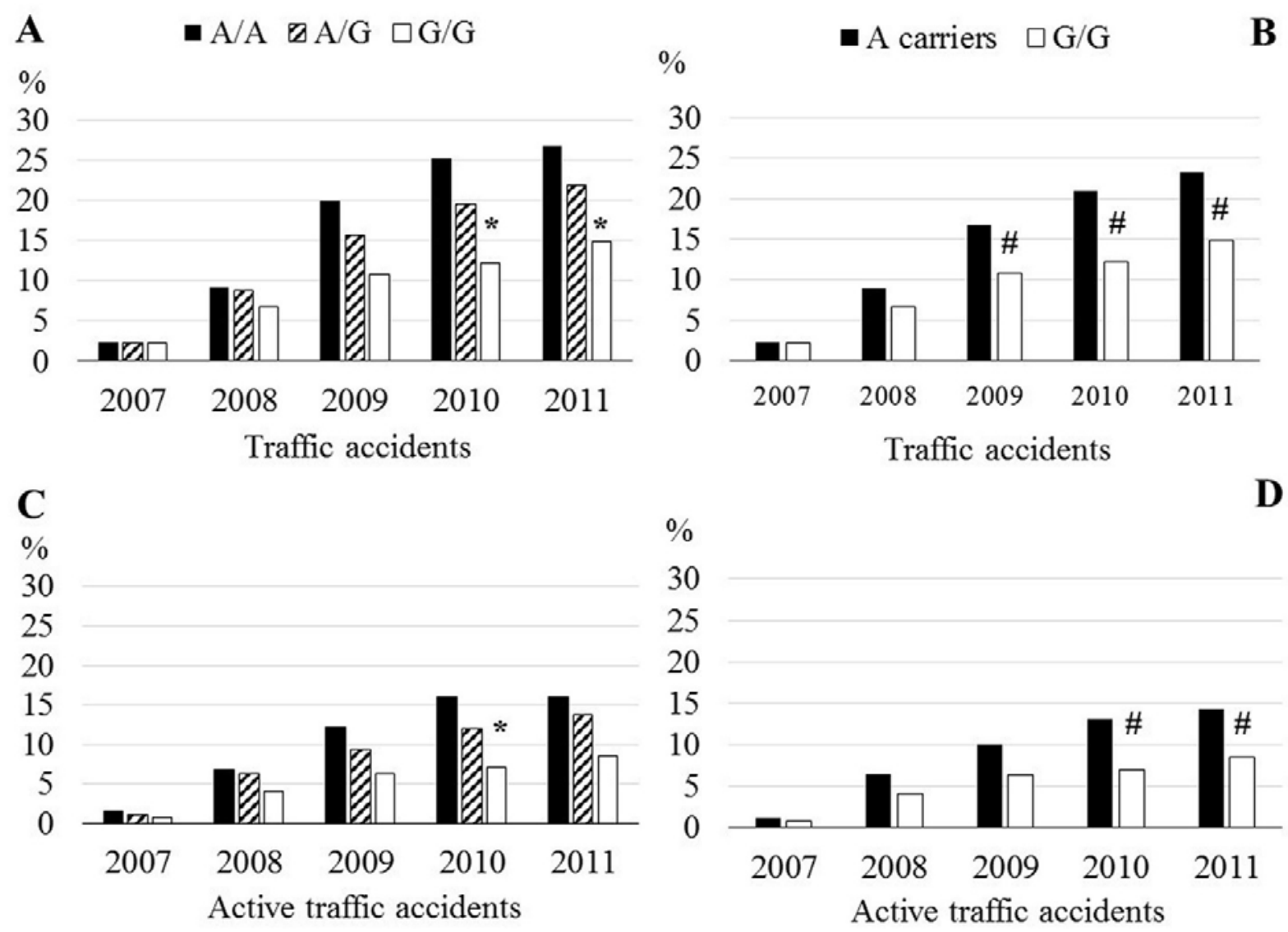

Fig. 6. Cumulative occurrence of total number (A and $B$ ) and active (C and D) traffic accidents in 2007-2011 by year in subjects recruited in 2007 at traffic schools, by the HCRTR1 genotype. The Yaxis represents the proportion of subjects with the given genotype who had by that year been involved in a traffic accident. * $-\mathrm{p}<0.05$, difference from the $\mathrm{A} / \mathrm{A}$ genotype; \# - difference from the A-allele carriers. Genotype frequency: $A / A=131, A / G=365, G / G=269$.

\section{Discussion}

In this study we have demonstrated that the orexin system is associated with aggressiveness and accident-prone behaviour in humans. Specifically, homozygocity for the minor variant of the HCRTR1 gene (rs2271933, G1222A) that causes amino acid substitution (Ile408Val) was associated with aggressiveness measured with either self-reports or clinical interviews. While the paper-and-pencil and interview measures of aggressiveness are in positive correlation (Kiive et al., 2017 and this study), these correlations in birth cohort representative samples are not particularly high. Nonetheless, the genotype effect was apparently very similar with both types of measurement, suggesting that the HCRTR1 genotype is related to aspects of 
aggressiveness that are represented equally in these two instruments. Importantly, the findings in the younger ECPBHS birth cohort, assessed for aggressiveness at age 25 in 2014/2015 were replicated in the older ECPBHS birth cohort assessed at age 33 in 2016/2017. It is of notice that genotype associations with behavioural measures in these two birth cohorts have not been similar in a variety of previous analyses. Indeed, for behaviours with strong secular changes such as age of alcohol use debut, striking genotype by birth cohort interactions have been revealed (Vaht et al., 2014, 2016). These may be related to changes in attitudes toward alcohol use in adolescents and alcohol availability during the societal changes that were very rapid in some of the countries of Central and Eastern Europe during the period when the two birth cohorts were in critical age (Harro and Vaht, 2019). The high similarity of the association of the HCRTR1 genotype with two different measures of aggressive behaviour in these two cohorts not only provides a necessary replication of the original observation but also suggests that the HCRTR1 genotype has an effect on aggressiveness that transcends such gene-environment effects that stem from societal changes.

Nevertheless, in females the HCRTR1 genotype effect was sensitive to stressful life events at the personal level. Again, the findings were similar with questionnaire and interview measures. Subjects with higher exposure to SLE in childhood had higher level of aggressiveness. This increase was small and not significant in female G/G homozygotes, and in the A/A males who had the highest levels of aggressiveness the stress exposure had not further effect, rather a nonsignificant trend towards reduction was observed. The interaction between genotype, SLE and sex was largely driven by the very large increase of aggressiveness in female A/A homozygotes with higher exposure to stress, while the female A/A homozygotes with low stress exposure did not differ from other genotypes. 
An initial attempt to understand the mediating mechanisms of the sex differences was made by examining the facets of the Affective Neuroscience Personality Scale in males and females. The ANPS (Davis and Panksepp, 2011) has been constructed bottom-up to measure the aspects of personality arising from persistent individual differences in the emotive phylogenetically old brain circuits and has been advocated for use in biologically oriented psychological studies (Montag and Reuter, 2014). Facets of the ANPS have been linked to characteristic patterns of resting state functional connectivity in the human brain (Deris et al., 2017). We expected to observe higher ANGER in both male and female HCRTR1 A/A homozygotes, and this was found indeed. Scores of FEAR were higher in females than males, and in particular in female A/A homozygotes. Female A/A homozygotes also had the lowest scores of SEEKING of all sex by genotype groups, while the A/A males had the highest. Altogether these findings lead to speculation that in male A/A homozygotes the relatively high aggressiveness levels that are not dependent of the experience of life adversities originate from higher activities of neural systems for ANGER and SEEKING, the latter promoting resource orientated behaviour. In female A/A homozygotes the high sensitivity to stressful life events may emerge from higher FEAR and low SEEKING. However, as aggressiveness and the personality facets were assessed cross-sectionally in adulthood, this hypothesis requires examination in further longitudinal studies.

It would be most important to know whether the higher aggressiveness in the HCRTR1 A/A homozygotes can lead to consequences in terms of real life behaviour. For this reason we genotyped the participants of a longitudinal study of traffic behaviour. Risky driving and traffic accidents are in strong relationship with aggressive and anxious states while driving, and share such affective and cognitive characteristics as emotional reactivity and threat appraisal (Zinzow and Jeffirs, 2018). We have previously shown in the EPSTB Sample 1 of this study that driving while impaired is highly recurrent, and that even in terms of other, 
additional traffic violations the repeat DWI drivers represent the most hazardous group of car drivers (Tokko et al., 2019). Driving while impaired is a high-risk behaviour that derives not just from the drinking problems but also temperament, personality, and development of basic behavioural tendencies (Stacy et al., 1991). The present study has found that recurrent DWI is more frequent in the HCRTR1 A/A homozygotes. Drunk driving was not more prevalent with this genotype in subjects with no record of major traffic violations, and if anything it was lower in drivers who had to a very significant extent exceeded speed limits. This suggests a rather specific relationship of HCRTR1 A/A homozygocity with drunk driving: It appears to aggravate problems with behavioural regulation in subjects who use alcohol irresponsibly. In the drunk drivers group the cumulative causing of traffic accidents was lower in HCRTR1 G/G homozygotes than in the A-allele carriers. Among the speed limit exceeders a rather different trend of the A/A homozygotes to cause less traffic accidents was revealed, but indeed the speed limit exceeders and drunk drivers have very different personality profiles (Paaver et al., 2006). We next examined the association of the HCRTR1 genotype with traffic accidents in a different sample of younger passenger car drivers that was recruited directly in traffic schools and consisted of both males and females. It was observed again that the genotype was associated with the active traffic accidents. The HCRTR1 A/A homozygotes had the highest cumulative accident rate of accidents, while the G/G homozygotes had the lowest. Given that the findings in these younger and less experienced car drivers resembled those obtained only in drunk drivers if the sample had longer driving records, it could be hypothetized that the HCRTR1 genotype relates to accident proneness in conditions of low behavioural control.

Previously, orexins have been implicated in coordinating defense response but mostly without focus on aggressiveness. However, one highly relevant study on epileptic patients monitored the release of orexin A in amygdala and found it to be increased while subjects 
reported higher levels of anger, but also more universally during social interactions; particularly high levels were observed during experiencing positive emotions (Blouin et al., 2013). Orexin A overflow was not related to arousal unspecifically, as it did not occur if arousal was related to postoperative pain. These findings in humans are consistent with data showing higher orexin neuron firing during operant reinforcement (Borgland et al., 2009; McGregor et al., 2011; Sharf et al., 2010). It is however not clear what is the respective contribution of $\mathrm{ORX}_{1}$ and $\mathrm{ORX}_{2}$ receptors in these responses. The $\mathrm{ORX}_{2}$ receptors were recently shown to contribute to emotion-related adaptive strategies dependent on environmental conditions (Staton et al., 2018).

To understand fully the neurobiological mechanisms behind the findings presented herewith, further information would be needed on the functional consequences of the HCRTR1 rs2271933 G > A variation. The G1222A variation leads to amino acid substitution in the region of receptor that may be interacting with $G$ proteins and other proteins (Thompson et al., 2014), but direct in vitro evidence of functionality is missing, while the valine in the respective position could promote the phosphorylation of the immediately adjacent serine and alter signal transduction upon activation of the receptor (Meerabux et al., 2005). The A-allele was found to be associated with higher prevalence of major depressive disorder (Rainero et al., 2011a), with a tendency for a larger difference from control with the A/A homozygotes than with A/G heterozygotes; however, the association of migraine with the HCRTR1 genotype rather rested on heterozygotes. These studies were not sufficiently large to suggest clearly the mode of inheritance. A recent study that separated migraine patients to those with aura and those without replicated the overrepresentation of the HCRTR1 A-allele in migraine patients without aura (Kowalska et al., 2018). The HCRTR1 genotype was not associated with narcolepsy in a small sample (Peyron et al., 2000) or with panic disorder (Annerbrink et al., 2011). Nevertheless, the presented findings should increase interest in elucidating the 
possible functional role of the HCRTR1 rs2271933 G > A polymorphism and other gene variations in the orexin signalling system to reveal the genetic mechanisms of the role of orexins in the coordinated defense response.

As a limitation of the presented study, the reader should be aware of the uncorrected p-values used throughout the text and illustrations. Given that the fair level of correction is not simple to select for the intercorrelated measures, and that it would put limits to relevant analyses given the fixed sample sizes of longitudinal studies, it was preferred to present all analyses as exploratory and leave the decision of the appropriate significance level with the reader. The strength of the study is inclusion of multiple cohorts and convergence of findings with related yet independent constructs.

Conclusively, we have found a consistent association of the HCRTR1 rs2271933 G > A variation with aggressiveness in two birth cohort samples. This gene polymorphism was also associated with high relapsing to drunk driving and with higher rate of causing traffic accidents among drivers with limited experience or hazardous driving behaviour. This evidence suggests on important role of orexins in the formation of aggressive responses and their control.

\section{Declarations of interest}

None.

\section{Acknowledgements}

We are grateful to the ECPBHS and EPSTB participants, and the respective study teams. This work was supported by Estonian Ministry of Education and Research Projects IUT20-40 and IUT42-2, the European Commission Seventh Framework Programme (FP7 2007-2013) Project Aggressotype ( $\left.{ }^{\circ} 602805\right)$, Horizon 2020 Programme Projects CoCA (n667302) and Eat2beNICE ( $\left.{ }^{\circ} 728018\right)$, and the Estonian Road Administration. 


\section{Appendix A.}

Supplementary data Supplementary data to this article can be found online at https://doi.org/10.1016/j.neuropharm.2019.02.009.

\section{References}

Annerbrink, K., Westberg, L., Olsson, M., Andersch, S., Sjödin, I., Holm, G., Allgulander, C., Eriksson, E., 2011. Panic disorder is associated with the Val308Iso polymorphism in the hypocretin receptor gene. Psychiatr. Genet. 21, 85-89.

Berridge, C.W., Espana, R.A., Vittoz, N.M., 2010. Hypocretin/orexin in arousal and stress. Brain Res. 1314, 91-102.

Blouin, A.M., Fried, I., Wilson, C.L., Staba, R.J., Behnke, E.J., Lam, H.A., Maidment, N.T., Karlsson, K.A., Lapierre, J.L., Siegel, J.M., 2013. Human hyprocretin and melanin-concentrating hormone levels are linked to emotion and social interaction. Nature Comm. 4, 1547.

Borgland, S.L., Chang, S.J., Bowers, M.S., Thompson, J.L., Vittoz, N., Floresco, S.B., Chou, J., Chen, B.T., Bonci, A., 2009. Orexin A/hypocretin-1 selectively promotes motivation for positive reinforcers. J. Neurosci. 29, 11215-11225.

Broberger, C., De Lecea, L., Sutcliffe, J.G., Hökfelt, T., 1998. Hypocretin/orexin- and melaninconcentrating hormone-expressing cells form distinct populations in the rodent lateral hypothalamus: Relationshipto the neuropeptide Y and agouti gene-related protein systems. J. Comp. Neurol. 402, 460-474.

Broberger, C., Hökfelt, T., 2001. Hypothalamic and vagal neuropeptide circuitries regulating food intake. Physiol. Behav. 74, 669-682.

Brundin, L., Björkquist, M., Petersen, A., Träskman-Bendz, L., 2007. Reduced orexin levels in the cerebrospinal fluid of suicideal patients with major depressive disorder. Eur.

Neuropsychopharmacol. 17, 573-579. 
Buss, A.H., Perry, M., 1992. The aggression questionnaire. J. Pers. Soc. Psychol. 63, 452-259.

Coccaro, E.F., Berman, M.E., Kavoussi, R.J., 1997. Assessment of life history of aggression: development and psychometric characteristics. Psychiatry Res. 73, 147-157.

D’Anna, K.L., Gammie, S.C., 2006. Hypocretin-1 dose-dependently modulates maternal behaviour in mice. J. Neuroendocrinol. 18, 553-566.

Davis, K.L., Panksepp, J., 2011. The brain’s foundations of human personality and the Affective Neuroscience Personality Scales. Neurosci. Biobehav. Rev. 35, 1946-1958.

Davis, K.L., Panksepp, J., Normansell, L., 2003. The affective neuroscience personality scales: Normative data and implications. Neuropsychoanalysis 5, 57-69.

de Lecea, L., Kilduff, T.S., Peyron, C., Gao, X., Foye, P.E., Danielson, P.E., Fukuhara, C., Battenberg, E.L., Gautvik, V.T., Bartlett, F.S. $2^{\text {nd }}$, Frankel, W.N., van den Pol, A.N., Bloom, F.E., Gautvik, V.T., Sutcliffe, J.G., 1998. The hypocretins: hypothalamus-specific peptides with neuroexcitatory activity. Proc. Natl. Acad. Sci. U.S.A. 95, 322-327.

Deris, N., Montag.C., Reuter, M., Weber, B., Markett, S., 2017. Functional connectivity in the resting brain as biological correlate of the Affective Neuroscience Personality Scales. NeuroImage 147, 423-431.

Dhabhar, F.S., 2018. The short-term stress response - Mother nature’s mechanism for enhancing protection and performance under conditions of threat, challenge, and opportunity. Front. Neuroendocrinol. 49, 175-192. https://doi.org/10.1016/j.yfrne.2018.03.004.

Eensoo, D., Paaver, M., Harro, M., Harro, J., 2005. Predicting drunk driving: contribution of alcohol use and related problems, traffic behaviour, personality and platelet monoamine oxidase (MAO) activity. Alcohol Alcohol. 40, 140-146.

Eensoo, D., Paaver, M., Vaht, M., Loit, H.-M., Harro, J., 2018. Risky driving and the persistent effect of a randomized intervention focusing on impulsivity: The role of the serotonin transporter promoter polymorphism. Accident Anal. Prev. 113, 19-24. 
Ferrario, C.R., Labouebe, G., Liu, S., Nieh, E.H., Routh, V.H., Xu, S., O’Connor, E.C., 2016. Homeostasis meets motivation in the battle to control food intake. J. Neurosci. 36, 11469-11481.

Flores, A., Saravia, R., Maldonado, R., Berrendero, F., 2015. Orexins and fear: implications for the treatment of anxiety disorders. Trends Neurosci. 38, 550-559.

Harro, J., Vaht, M., 2019. Molecular genetics meets sociology: Birth cohort effects on alcohol use and relationship with candidate genes, in: Preedy, V.M. (Ed.), Neuroscience of Alcohol. Elsevier, in press. DOI: https://doi.org/10.1016/B978-0-12-813125-1.00002-7.

Johnson, P.L., Molosh, A., Fitz, S.D., Truitt, W.A., Shekhar, A., 2012. Orexin, stress, and anxiety/panic states. Prog. Brain Res. 198, 133-161.

Kiive, E., Laas, K., Vaht, M., Veidebaum, T., Harro, J., 2017. Stressful life events increase aggression and alcohol use in young carriers of the GABRA2 rs279826/rs279858 A-allele. Eur. Neuropsychopharmacol. 27, 816-827.

Kowalska, M., Kapelusiak-Pielok, M., Grzelak, T., Wypasek, E., Kozubski, W., Dorszewska, J., 2018. The new *G29A and G1222A of HCRTR1, 5-HTTLPR of SLC6A4 polymorphisms and hypocretin-1, serotonin concentrations in migraine patients. Front. Mol. Neurosci. 11, 191. DOI: 10.3389/fnmol.2018.00191.

Kruk, M., 2014. Hypothalamic attack: A wonderful artifact or a useful perspective on escalation and pathology in aggression? A viewpoint. Curr. Top. Behav. Neurosci. 17, 143-188.

Laas, K., Kiive, E., Mäestu, J., Vaht, M., Veidebaum, T., Harro, J., 2017. Nice guys: Homozygocity for the TPH2 -703G/T (rs4570625) minor allele promotes low aggressiveness and low anxiety. J. Affect. Disord. 251, 230-236.

Laas, K., Reif, A., Kiive, E., Domschke, K., Lesch, K.P., Veidebaum, T., Harro, J., 2014. A functional NPSR1 gene variant and environment shape personality and impulsive action: A longitudinal study. J. Psychopharmacol. 28, 227-236. 
McGregor, R., Wu, M.-F., Barber, G., Ramanathan, L., Siegel, J.H., 2011. Highly specific role of hypocretin (orexin) neurons: Differential activation as a function of diurnal phase, operant reinforcement versus operant avoidance and light level. J. Neurosci. 31, 15455-15467.

Meerabux, J., Iwayama, Y., Sakurai, T., Ohba, H., Toyota, T., Yamada, K., Nagata, R., IrukayamaTomobe, Y., Shimizu, H., Yoshitsugu, K., Ohta, K., Yoshikawa, T., 2005. Association of an orexin 1 receptor $408^{\text {Val }}$ variant with polydipsia-hyponatremia in schizophrenic subjects. Biol. Psychiat. 58, 401-407.

Montag, C., Reuter, M., 2014. Disentangling the molecular genetic basis of personality: From monoamines to neuropeptides. Neurosci. Biobehav. Rev. 43, 228-239.

O’Connor, B.O., Archer, J., Wu, F.W.C., 2001. Measuring aggression: Self-reports, partner reports, and responses to provoking scenarios. Aggr. Behav. 27, 79-101.

Paaver, M., Eensoo, D., Kaasik, K., Vaht, M., Mäestu, J., Harro, J., 2013. Preventing risky driving: A novel and efficient brief intervention focusing on acknowledgement of personal risk factors. Accident Anal. Prev. 50, 430-437.

Paaver, M., Eensoo, D., Pulver, A., Harro, J., 2006. Adaptive and maladaptive impulsivity, platelet monoamine oxidase (MAO) activity and risk-admitting in different types of risky drivers. Psychopharmacology 186, 32-40.

Panksepp, J., 1998. Affective Neuroscience: The Foundations of Human and Animal Emotions. Oxford University Press.

Peyron, C., Faraco, J., Rogers, W., Ripley, B., Overeem, S., Charnay, Y., Nevsimalova, S., Aldrich, M., Reynolds, D., Albin, R., Li, R., Hungs, M., Pedrazzoli, M., Padigaru, M., Kucherlapati, M., Fan, J., Maki, R., Lammers, G.J., Bouras, C., Kucherlapati, R., Nishino, S., Mignot, E., 2000. A mutation in a case of early onset narcolepsy and a generalized absence of hypocretin peptides in human narcoleptic brains. Nature Med. 6, 991-997. 
Rainero, I., Ostacoli, L., Rubino, E., Gallone, S., Picci, L.R., Fenoglio, P., Negro, E., Rosso, C., De Martino, P., De Marchi, M., Furlan, P.M., Pinessi, L., 2011a. Association between major mood disorders and the hypocretin receptor 1 gene. J. Affect. Disord. 130, 487-491.

Rainero, I., Rubino, E., Gallone, S., Fenoglio, P., Picci, L.R., Giobbe, L., Ostacoli, L., Pinessi, L., 2011b. Evidence for an association between migraine and the hypocretin receptor 1 gene. J. Headache Pain 12, 193-199.

Sakurai, T., Amemiya, A., Ishii, M., Matsuzaki, I., Chemelli, R.M., Tanaka, H., Williams, S.C., Richardson, J.A., Kozlovski, G.P., Wilson, S., Arch, J.R., Buckingham, R.E., Haynes, A.C., Carr, S.A., Annan, R.S., McNulty, D.E., Liu, W.S., Terrett, J.A., Elshourbagy, N.A., Bergsma, D.J., Yanagisawa, M., 1998. Orexins and orexin receptors: a family of hypothalamic neuropeptides and G protein-coupled receptors that regulate feeding behavior. Cell 92, 573-585.

Sargin, D., 2018. The role of the orexin system in stress response. Neuropharmacology (in press). DOI: 10.1016/j.neuropharm.2018.09.034.

Schwartz, M.D., Kilduff, T.S., 2015. The neurobiology of sleep and wakefulness. Psychiatr. Clin. North Am. 28, 615-644.

Sharf, R., Sarhan, M., Brayton, C.E., Guarnieri, D.J., Taylor, J.R., DiLeone, R.J., 2010. Orexin signalling via the orexin 1 receptor mediates operant responding for food reinforcement. Biol. Psychiatry 67, 753-760.

Stacy, A.W., Newcomb, M.D., Bentler, P.M., 1991. Personality, problem drinking, and drunk driving: Mediating, moderating, and direct-effect models. J. Pers. Soc. Psychol. 60, 795-811.

Staton, C.D., Yaeger, J.D.W., Khalid, D., Haroun, F., Fernandez, B.S., Fernandez, J.S., Summers, B.K., Summers, T.R., Sathyanesan, M., Newton, S.S., Summers, C.H, 2018. Orexin 2 receptor stimulation enhances resilience, while orexin 2 inhibition promotes susceptibility, to social stress, anxiety and depression. Neuropharmacology 143, 79-94. 
Sutcliffe, J.G., de Lecea, L., 2000. The hypocretins: Excitatory neuromodulatory peptides for multiple homeostatic systems, including sleep and feeding. J Neurosci. Res. 62, 161-168.

Thompson, M.D., Xhaard, H., Sakurai, T., Rainero, I., Kukkonen, J.P., 2014. OX ${ }_{1}$ and $\mathrm{OX}_{2}$ orexin/hypocretin receptor pharmacogenetics. Front. Neurosci. 8, 57.

Tokko, T., Eensoo, D., Vaht, M., Lesch, K.P., Reif, A., Harro, J., 2019. Relapse of drunk driving and association with traffic accidents, alcohol-related problems, and biomarkers of impulsivity. Acta Neuropsychiat., in press.

Van Kampen, H.S., 2015. Violated expectancies: cause and function of exploration, fear, and aggression. Behav. Processes 117, 12-28.

Zinzow, H.M., Jeffirs, S.M., 2018. Driving aggression and anxiety: Intersections, assessment, and interventions. J. Clin. Psychol. 74, 43-82.

Vaht, M., Kiive, E., Veidebaum, T., Harro, J., 2016. A functional vesicular monoamine transporter 1 (VMAT1) gene variant is associated with affect and the prevalence of anxiety, affective, and alcohol use disorders in a longitudinal population-representative birth cohort study. Int. J. Neuropsychopharmacol. 19, pyw013.

Vaht, M., Merenäkk, L., Mäestu, J., Veidebaum, T., 2014. Serotonin transporter gene promoter polymorphism (5-HTTLPR) and alcohol use in general population: Interaction effect with birth cohort. Psychopharmacology 231, 2587-2594. 